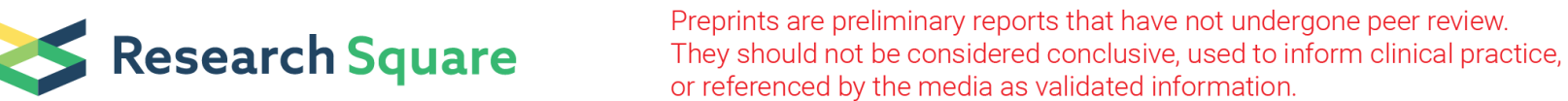

\section{Corneal reconstruction in chemically damaged cornea using temperature sensitive surface assisted mesenchymal stem cell transplantation in rabbits}

Nirbhai Singh ( $\nabla$ nirbhais@gmail.com )

Post Graduate Institute of Medical Education and Research https://orcid.org/0000-0002-5449-1330

Jyoti Sharma

Post Graduate Institute of Medical Education and Research

Pankaj Gupta

Post Graduate Institute of Medical Education and Research

Ravi Kumar Sharma

Post Graduate Institute of Medical Education and Research

Nalini Gupta

Post Graduate Institute of Medical Education and Research

\section{Research article}

Keywords: Chemical corneal damage, MSC transplantation, Temperature sensitive membranes, vimentin, collagen-1

Posted Date: February 21st, 2019

DOI: https://doi.org/10.21203/rs.2.371/v1

License: (c) (i) This work is licensed under a Creative Commons Attribution 4.0 International License.

Read Full License 


\section{Abstract}

Background: The study aims to assess the potential of corneal reconstruction using mesenchymal stem cells (MSCs) transplantation using temperature sensitive membranes as carriers and compare with fibrin glue method in chemically damaged rabbit cornea. Methods: MSCs were cultured from rabbit's bone marrow and transplanted over rabbit's cornea, using either temperature sensitive membranes or fibrin glue method. Endogenous levels of MSCs were assessed to decide the optimal time point for transplanting cells. MSC transplanted corneas were harvested 15, 30 and 60 days post transplantation. Corneal repair markers were evaluated using histopathology, immunohistochemistry (IHC) and real time PCR. The quality of cornea reconstructed was evaluated using corneal opacity scoring and IHC. Results: Endogenous levels of MSCs were significantly higher 48 hours post corneal injury. Transplantation of MSCs using temperature sensitive method resulted in uniform and homogenous spread of MSCs over the damaged surface. Corneal transparency improved 7 days onward in rabbit chemical injured cornea after MSC transplantation. Immunohistochemistry (IHC) of damaged cornea showed complete absence of corneal epithelium as seen by CK3 expression, while re-epithelialization was observed after MSC transplantation. Histopathology showed complete re-epithelialization 15 days of transplantation. Evaluation of corneal quality using epithelial and stromal markers showing restoration of vimentin, asmooth muscle actin and collagen levels in MSC transplanted cornea compared to damaged corneas. CK3 expression showed differentiation of MSC into corneal epithelial cells. MSC transplanted corneal clarity seemed a little better with temperature sensitive method compared to fibrin glue method. No toxic effects of MSC transplantation were observed on other organs or on resident corneal cells. Conclusions: Temperature sensitive surface MSC transplantation method results in uniform and homogenous delivery of cells on corneal surface and a transparent clear cornea. Epithelial and stromal markers showing restoration of vimentin, a-smooth muscle actin and collagen levels in MSC transplanted cornea compared to chemically damaged corneas. Our data also show the differentiation of MSC into corneal epithelial cells on corneal surface. Overall temperature surface MSC transplantation is an easy and safe alternative method to fibrin glue for exploring corneal reconstruction.

\section{Background}

Corneal blindness is the second leading cause of blindness with prevalence of 4.9 million cases across the globe [1]. In case of insult to the cornea, limbal stem cells in the basement kick start the repair and maintain corneal transparency by various cytoskeletal elements including vimentin, alpha smooth muscle actin and collagen-1 [2]. Common treatment modalities in severe chemical injuries include the use of amniotic membranes, autologous serum and bandage contact lenses, with variable scales of success [35]. Transplantation strategies include autologous limbal grafts in case of unilateral limbal stem cell deficiency (LSCD) or unilateral injury, while limbal allografts in bilateral LSCD. However the grafts involving allogenic limbus transplantation significantly increase the chances of graft rejection as compared to corneal transplant [6]. 
These challenges have resulted in focus on stem cell research towards corneal reconstruction [7-12]. Hematopoietic stem cells and mesenchymal stem cells (MSCs) have primarily drawn attention and tried by several groups in corneal reconstruction. MSCs being non-hematopoietic and self-renewing cells with potency and differentiation potential comparable to embryonic stem cells [13]. Additionally, MSCs have been known to exert anti-inflammatory and anti-angiogenic effects on the cornea, favouring the therapeutic potential of MSCs transplantation [11]. Various carriers including amniotic membranes, temperature responsive surfaces and fibrin glue have been explored for cell transplantation over damaged corneal surface [14-18].

MSCs have been shown the potential to differentiate into corneal epithelial cells [9]. These cells were effectively able to reconstruct corneal surface in an alkali injury rat model using amniotic membranes [10]. Also, MSCs or their conditioned media applied over injured cornea, showed significant corneal wound healing [19]. Despite these reports on feasibility of MSCs as choice of transplantation, there are scanty reports of MSC transplantation using temperature sensitive surfaces and no study to the best of our knowledge have compared the efficacy of temperature sensitive surfaces with routinely used methods of cell transplantation for corneal reconstruction.

In view of these reports, we aimed to assess the feasibility and efficacy of temperature responsive surfaces for transplantation of MSCs. We attempted to transplant autologous MSCs over damaged cornea of New Zealand White rabbits, using either a temperature sensitive surface or fibrin glue assisted transplantation. Rabbit was chosen due to its similarities of corneal parameters such as endothelial cell density, central corneal thickness, decrease in corneal diameter with age, to that of humans. MSCs were transplanted at the time showing highest endogenous levels of MSCs in peripheral blood. Corneal repair and quality of cornea reconstructed was assessed by clinical scoring, OCT Imaging and IHC using corneal and stromal markers. Differentiation of MSC into corneal epithelial markers was evaluated using CK3 expression.

\section{Methods}

\section{Corneal injury model:}

Rabbits were subjected to corneal injury under general anaesthesia with an intramuscular injection of Xylazine $(12 \mathrm{mg} / \mathrm{kg})$ and ketamine $(40 \mathrm{mg} / \mathrm{kg})$ during the day. Corneal wounds were made by contact with filter paper ( $15 \mathrm{~mm}$ diameter; 30 s contact time) soaked in $1 \mathrm{~N} \mathrm{NaOH}$, followed by corneal and limbal epithelium scrapping using a surgical blade. Antibiotic drops (Moxifloxacin 0.5\%) were applied four times a day for 7 days. No immunosuppressive drugs were used in the study. Fluorescein staining was performed to evaluate the degree of corneal damage.

MSC isolation and culture: Rabbits were euthanized with overdose of barbiturates i.e. thiopentone $(20 \mathrm{mg} / \mathrm{kg})$. A cut around tibia and femur was made aseptically and the bones were collected in low glucose DMEM medium with 1\% penicillin-streptomycin, kept on ice. Approximately $8 \mathrm{ml}$ of marrow tissue was aspirated using biopsy needle (18 gauge) and poured in a sterile petri dish. Tissue was chopped 
finely and small pieces were placed in 24 well cell culture plates with media just covering the tissue. Culture media $(500 \mu \mathrm{L})$ was added into each well after 2 days of undisturbed incubation at $37^{\circ} \mathrm{C}, 5 \% \mathrm{CO}_{2}$. The adherence and confluency was assessed and subcultures performed periodically for 14 days. After the cells attained confluency they were transferred to T-25 flask. Trypsinization conditions were kept such that there was minimum contamination by non-MSCs $\left(2\right.$ mins; $\left.25^{\circ} \mathrm{C}\right)$ as reported earlier. ${ }^{[20]}$

\section{Purity assessment and characterization of cultured MSCs:}

The cultured cells were morphologically assessed using microscopy. The cells were then characterized for the expression of MSC markers including CD90, CD81, CD34 and HLA-DR using anti rabbit CD90-FITC (OX-7), CD34 (QBEnd-10) (primary antibody) (Thermo Fisher Scientific, MO, USA) and anti mouse IgG1PerCP (secondary antibody) (BD Biosciences, CA, USA), anti rabbit CD81-APC (JS-81) and anti human HLA-DR-PE (TU36) (BD) by flow cytometry. Briefly, $10^{6}$ cells were stained with $5 \mu$ l of anti rabbit CD34 antibody per tube and incubated for 30 minutes at room temperature (RT), followed by washing with $1 \mathrm{X}$ PBS. The washed cells were then stained with anti mouse IgG1 Per-CP and incubated at RT for 30 minutes. The cells were washed with 1X PBS and stained with anti rabbit CD90-FITC, CD81-APC and HLADR-PE antibodies, followed by washing and acquisition on flow cytometer and data analysed using FACS Diva software version 7.0. Cells were gated on the basis of forward and side scatter profile, followed by doublet discrimination and gating of assessment of proportion of MSCs in the gated cluster. The purity of CD $90^{+}$CD $81^{+}$CD34 HLA-DR ${ }^{-}$MSCs was calculated. Hoechst pumping assay was also employed for characterization of MSCs [21]. Briefly, the MSCs cultured over cover slip were stained using Hoechst 33342 dye (1:2,000 of $10 \mathrm{mg} / \mathrm{ml}$ stock) (Thermo). Blue and red Hoechst fluorescence signals were collected with 424/44 band pass and 620 long pass filter respectively, displayed on a linear, dualfluorescence dot plot. The data was acquired on FACS LSR Fortessa flow cytometer (BD) and analysed using FACS Diva Software v 7.0.

\section{Endogenous levels of MSCs and chemoattractants:}

Ear vein puncture was performed to withdraw peripheral blood at $0,24,48,72$ hours post corneal injury and blood collected in EDTA and serum vials. Serum was preserved at $-80^{\circ} \mathrm{C}$ for ELISA. The frequencies of MSCs were assessed in cells using flow cytometry as discussed above. The frequencies of MSCs were reported as percent of gated cells (in region defined for MSCs on the basis of forward and side scatter). The Levels of SDF-1 and substance P (chemoattractants) in serum were analysed using Rabbit Stromal Cell Derived Factor 1 alpha (SDF-1a) ELISA kit \& Substance P ELISA kit (Sincere Biotech Co., Ltd, Beijing, China) assay kit, as per manufacturer prescriptions. The minimum detection limit (with intra, inter assay CV) for SDF1-a was $31.1 \mathrm{pg} / \mathrm{mL}(\leq 9.00, \leq 15.0 \%)$ and that of substance P was $12.6 \mathrm{pg} / \mathrm{mL}(\leq 9.00$, $\leq 15.0 \%$ ). All the samples were run in duplicates and the concentration of each cytokine was interpolated from standard curves using standards provided with the kits.

MSC transplantation: MSCs were transplanted 48 hours post corneal injury (Table 1). Prior to transplantation, MSCs were labelled with CFSE for assessing the distribution and retention of MSCs over 
the damaged corneal surface. In one set of experiments, MSCs were cultured overnight, in temperature sensitive culture plates (Nunc UpCell Surface, Roskilde, Denmark), followed by placement of the membrane over the cell layer at $20^{\circ} \mathrm{C}$ for 15 minutes for facilitating the transfer of MSCs onto the membrane. The membrane was then placed over the damaged cornea and kept for 30 minutes to allow the adherence of cells to the corneal surface. In independent experiments, MSCs were applied drop wise $\left(2 \times 10^{5} \mathrm{cells} / \mathrm{ml}\right)$ along with fibrin glue (sealer protein and thrombin solution) over the damaged cornea (Baxter International, IL, USA). No immunomodulatory drugs were used during or after transplantation. Rabbit corneas were imaged weekly, a group of rabbits were sacrificed at day 15,30 and day 60 .

Fluorescein staining: Fluorescein staining was performed on corneas pre and post injury to ensure complete corneal damage after injury and before enucleation for assessing the effect of transplantation on healing of epithelium. Briefly fluorescein strips were placed between eyelids of animals, after local anaesthesia using proparacaine and eyes washed with normal saline. Images of eye were taken using a DSLR camera (Canon Inc., Japan). The extent of epithelial defect was compared between 4 groups at all time points by scoring the images using clinical scoring criteria [22].

OCT Imaging: Rabbits were anesthetized by lower dose of Xylazine $(10 \mathrm{mg} / \mathrm{kg})$ and ketamine $(35 \mathrm{mg} / \mathrm{kg})$ and subjected to anterior segment OCT after hydrating cornea using normal saline. Anterior segment OCT was then evaluated for corneal surface regularity and contour.

Confocal microscopy: The distribution of transplanted CFSE labelled MSCs over the damaged corneal surface was assessed using confocal microscopy of slides prepared after the enucleation. Briefly, the cornea was chopped to small pieces and placed over slides. The slides were then fixed and observed under confocal microscope in FITC channel.

Histopathology: Eyes were enucleated, washed with 1X PBS, followed by dissection of the cornea. The corneas were fixed in $4 \%$ paraformaldehyde (PFA) for 3 days. $4 \mu \mathrm{m}$ paraffin embedded sections were prepared, stained with Haematoxylin and eosin and observed under light microscope for qualitative evaluation (Olympus, Tokyo, Japan).

Immunohistochemistry: In order to evaluate epithelial and stromal markers in transplanted cornea, IHC was performed. Briefly, Four $\mu \mathrm{m}$ sections obtained from the paraffin-embedded cornea were transferred onto poly-L-Lysine coated slides (Sigma Aldrich). The antigen retrieval was performed by heat mediated antigen retrieval using sodium citrate buffer $(\mathrm{pH}=6)$. The Endogenous peroxidase activity was quenched by incubating the sections in 3\% hydrogen peroxide for 20 mins at RT. Subsequently, the slides were stained with primary anti-rabbit cytokeratin 3 (1:80), vimentin (1:20), alpha SMA (1:150) and Collagen 1 (1:350) antibodies (Thermo) overnight at $4^{0} \mathrm{C}$ followed by washing with PBS. The slides were then stained with HRP conjugated secondary goat anti-rabbit IgG antibody for 1 hour at RT, followed by addition of diaminobenzidine (DAB) solution. The slides were counter stained with haematoxylin and mounted with DPX mountant (Sigma Aldrich). The slides were then viewed under light microscope at 20X 
magnification (Olympus BX53, Tokyo, Japan). Negative controls were performed in the absence of primary antibodies.

TUNEL ASSAY: The extent of apoptosis was evaluated in corneal sections using tunnel assay kit, as per manufacturer instructions (TACS ${ }^{\circledR} 2$ TdT DAB kit, Trevigen, MD, USA). Briefly, the slides were deparaffinised, rehydrated and then treated with proteinase $K$, followed by blocking using $3 \%$ hydrogen peroxide. The slides were then labelled with the provided labelling buffer and stained with HRP and developed using DAB. The slides were then counterstained with methyl green. Positive control slides were made using TACS nuclease enzyme provided in the kit.

Statistical analysis: The frequency of endogenous MSCs and levels of chemoattractants were compared using one-way analysis of variance (ANOVA). Corneal grades and IHC scores were compared using MannWhitney $U$ test. The data was analysed using graphpad prism version 5.0. The data is depicted as Mean \pm SEM.

\section{Results}

Explant based culture from after bone marrow harvesting provides a homogenous population of MSCs: MSCs (Rb-MSCs) were cultured from rabbit bone marrow using explant-based approach. Trypsinization was performed $3^{\text {rd }}$ day after culturing and colonies of MSCs were observed in the culture dish after 5 days. The characteristic spindle shaped morphology was clearly observed after 5 days of explant culture (Figure 1A). A single confluent layer of adherent cells was obtained after 14 days (Figure 1B). The cells from primary culture were trypsinised and characterised using rb-MSC markers i.e. CD90, CD81, CD34 and HLA-DR. MSC cluster was gated on the basis of forward and side scatter (Figure $1 \mathrm{C}$ ). $\mathrm{CD} 90^{+} \mathrm{CD} 81^{+}$cells

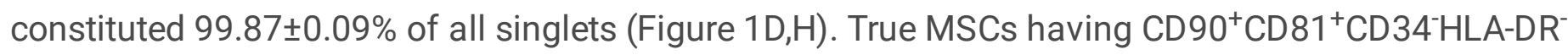
phenotype were found to be $94.13 \pm 2.03 \%$ of $\mathrm{CD} 81^{+} \mathrm{CD} 90^{+}$singlets (Figure $1 \mathrm{E}, \mathrm{H}$ ).

To further confirm the identity of cultured cells, these were subjected to hoechst staining. Hoechst 33342 dye stains fibroblasts whereas stem cells pump out this dye because of excessive sodium ATPase activity. We observed $81.10 \pm 1.94 \%$ of cultured cells to be showing the pumping activity for Hoechst 33342 (Figure 1F,G,H). Hoechst staining and immunophenotyping established that primary culture was a pure culture of MSCs.

\section{Chemical corneal injury increases endogenous levels of MSCs and chemoattractants promoting their} migration: To assess the effect of corneal injury of endogenous MSCs mobilization, we measured the frequencies of MSCs in the peripheral blood at 0 (Figure 2A), 24 (Figure 2B), 48 (Figure 2C) and 72 hours (Figure 2D) post corneal injury. There was an insignificant increase in frequency of endogenous MSCs in blood at 24 hours $(0.46 \pm 0.09 \%)$ as compared to frequency of MSCs before corneal injury $(0.41 \pm 0.09 \%)$ $(p=0.76, n=8)$ (Figure 2E). However, we found that the frequency of MSCs was significantly higher 48 hours after injury $(1.765 \pm 0.36 \%)$ as compared to that before injury $(p=.0019, n=8)$ (Figure 2E). These frequencies sustained till 72 hours, with levels being significantly higher $(0.99 \pm 0.36 \%)$ as compared to 
those before injury $(p=0.038, n=8)$. The levels of SDF1-a were found to be apparently higher 48 hours post injury $(384.1 \pm 54.32 \mathrm{pg} / \mathrm{ml})$ as compared to levels before injury $(252.3 \pm 15.68 \mathrm{pg} / \mathrm{ml})(\mathrm{p}=0.059, \mathrm{n}=4)$ (Figure $2 F, G)$. The elevated levels of substance $P 48$ hours post corneal injury $(115.60 \pm 2.74 \mathrm{pg} / \mathrm{ml})$ as compared to before injury $(85.76 \pm 4.95 \mathrm{pg} / \mathrm{ml})(\mathrm{p}=0.03, \mathrm{n}=4)($ Figure $2 \mathrm{H}, \mathrm{I})$ also indicated the mobilization of stem cell pool. There was no significant difference in levels post 24 or 72 hours. Taken together, these observations suggest that corneal injury does have a significant impact on mobilization of MSCs in the blood and number of MSCs vary at different time intervals after the corneal injury.

\section{MSC transplantation using temperature sensitive surfaces result in uniform spread of cells over damaged} surface: The healthy cornea didn't show any fluorescence i.e. grade 0 cornea (Figure 3A) while damaged rabbit cornea showed complete uptake of fluorescein dye i.e. grade 4 cornea (Figure 3B). Further damaged cornea was evaluated at different time points and showed ulcer formation by day 60 in the damage group (Figure 3C), while a clearer cornea was observed after MSC transplantation using temperature sensitive membranes (Figure 3D). Haematoxylin \& Eosin staining of normal cornea showed five layers of healthy cornea (Figure $3 \mathrm{E}$ ) while damage was confirmed by the partial or complete absence of corneal epithelium, migration of leucocytes and presence of RBCs (Figure 3F). The transplantation of MSCs using temperature sensitive surfaces (Figure $3 \mathrm{H}, \mathrm{J}$ ) resulted in a better and uniform spread of MSCs as compared to fibrin glue method (Figure 3G,I), as assessed by confocal microscopy (Olympus, Tokyo, Japan).

\section{Temperature sensitive surface assisted MSC transplantation over damaged cornea results in transparent} corneal surface: Corneal repair was assessed post MSC transplantation using various methods. Overall, we observed apparently better corneal quality using temperature sensitive surfaces as compared to fibrin glue assisted transplantation.

Corneal grading was performed in live rabbits by staining corneas using akorn ful-Go ${ }^{\circledR}$ fluorescein strips (Akorn Pharmaceuticals, IL, USA) and were clinically scored [22]. The scores were compared at different time points (15, 30 and 60 days) to damage alone cornea, in both treatment sets. The scores were found to be significantly higher in damaged group $(2.78 \pm 0.15)$ as compared to fibrin glue transplanted group $(0.56 \pm 0.17)(p=0.008, n=9)$ (Figure $4 A)$, as well as temperature sensitive membrane method $(0.33 \pm 0.17)$ $(p=0.008, n=9)$ (Figure 4B) on day 60 . It clearly showed significant healing of corneas with both methods in MSC transplanted group as compared to the chemically damaged cornea group. Temperature sensitive membrane assisted MSC transplantation showed apparently lower scores than that using fibrin glue $(p=0.07, n=9)$ (Figure 4C), indicating better efficiency using this method.

OCT imaging showed irregular cornea with no epithelial membrane in case of chemically damaged cornea group (Figure 4D,G), however chemically damaged rabbit corneal surface transplanted with MSC using both fibrin glue and temperature sensitive method resulted in epithelial membrane regeneration 15 day onward and transformed into smooth corneal surface (Figure 4E,H) and 30 days (Figure 4F,I). Temperature sensitive surfaces treated corneas appeared to have a little better corneal grading compared to fibrin glue methods however the difference was not found statistically significant. 
H\&E staining of corneal sections clearly showed damaged cornea with complete absence of cornea; epithelium, migration of leucocytes and RBCs (Figure $4 \mathrm{~J}$ ). while re-epithelialization of corneal epithelium was observed post transplantation using temperature sensitive membrane (Figure 4K) as well as fibrin glue approach (Figure 4L). Corneal stroma thickness which was increased post injury came back to its normal state after MSC transplantation.

Immunohistochemistry of CK3 further confirmed the re-epithelialisation and differentiation of MSC into corneal epithelial cells. The chemically damaged cornea resulted in loss of CK3 expression (Figure 4M) in corneal sections, however restored CK3 expression observed with both methods of MSC transplantation. Expression of CK3 was observed as early as 15 days post transplantation using both the approaches (Figure 4N,0).

\section{MSC transplantation restores corneal homeostasis for corneal reconstruction markers:}

Immunohistochemical studies were performed for corneal reconstruction markers including Collagen 1, alpha SMA and Vimentin [2]. There was a statistically significant increase in vimentin expression in cornea upon chemical induced damage $(p=0.013, n=3)$. MSC transplantation reversed the increase and we observed a statistically significant decrease in vimentin levels as compared to damaged corneas, $(p=0.02, n=3)$ (Figure 5 A-C,J). As expected there was no statistically significant difference in vimentin expression levels of control and transplanted cornea $(p=0.51, n=3)$.

We observed a statistically significant increase in expression of a-SMA upon corneal damage $(p=0.002$, $n=3)$. The expression pattern was reversed in eyes which received transplanted MSCs $(p=0.007, n=3)$. As observed for vimentin, the expression levels of a-SMA were not significantly different between control and transplanted cornea $(p=0.10, n=3)$ (Figure $5 D-F, K)$.

The comparison of control and damaged cornea showed an insignificant increase in collagen 1 expression levels $(p=0.10, n=3)$. We found a statistically significant increase in levels of collagen 1 in transplanted cornea as compared to damage alone group $(p=0.04, n=3)$. The comparison of control and transplanted cornea showed a statistically significant increase in collagen 1 levels in transplanted group, thereby signifying MSC induced wound healing ( $p=0.008, n=3)$ (Figure $5 \mathrm{G}-\mathrm{I}, \mathrm{L})$.

MSC transplantation doesn't induces apoptosis in neighbouring cells: Histology study of organs like kidney liver and spleen showed that there was no cell mass seen in these organs post transplantation (Figure $6 \mathrm{~A}, \mathrm{~B}$ ). No apoptosis in the surrounding cells was observed post MSC transplantation and control cornea (Figure $6 \mathrm{C}, \mathrm{E}, \mathrm{D})$.

\section{Discussion}

Commonly used methods for corneal stem cell transplantation include the use of amniotic membranes, contact lenses and fibrin glue with its own limitations [3, 5, 23]. A few studies have previously shown MSCs ability to differentiate into cells with morphology and phenotype similar to corneal epithelial cells $[9,10]$. MSCs cultured over amniotic membranes have been shown to reconstruct the damaged corneal 
surface via inhibition of inflammation. Systemic transplantation of MSCs also improved the corneal quality after chemical induced damage [24]. Bone marrow derived mesenchymal stem cells (MSCs) on amniotic membrane have been shown to trans-differentiate into corneal epithelial like cells in vitro [12, 25]. Human or rabbit limbal stem cells have been successfully cultured on temperature-responsive culture surfaces but not well characterized towards corneal cell phenotype $[17,26]$. Human undifferentiated immature dental pulp stem cells and autologous oral mucosal epithelial cells expanded ex vivo on temperature-responsive cell culture surfaces been shown for reconstruction of ocular surfaces without characterizing the repaired cornea cell type [26,27]. We have not only used temperature sensitive surfaces for corneal reconstruction, further have well characterized the MSC differentiation into corneal epithelial cells and finally compared with traditionally used fibrin glue method. Moreover, we performed extensive expression analysis of various molecules including vimentin, a-SMA, collagen-1, CK3 and performed OCT to check quality of reconstructed corneal surface and assessed long term impact of MSC transplantation over the damaged cornea. Our results clearly showed that quality of reconstructed cornea gradually improved in group of animals observed for 60 days as compared to 30 and 15 day groups. Corneal clarity was apparently better with temperature surface method compared to fibrin glue.

A major drawback with fibrin glue method of transplantation of MSCs or any other cells is clumping of cells and non-uniform layering over the damaged corneal surface. Temperature sensitive surfaces solve this problem by providing a single layer of cells with uniform cell distribution over damaged area. Cell sheet engineering method is been widely explored for tissue reconstruction in various organs [28, 29]. This method is better than injecting cells because of ease of controlling size, shape and location of grafted cells [28]. In our study, the delivery of cells appeared to be more uniform compared to fibrin glue, which showed continuous sheets of CFSE labelled MSCs while using temperature sensitive surfaces. Being a sutureless method, it is as safe as fibrin glue assisted transplantation. Further, these sheets allow cultured MSCs to interact immediately and directly with the damaged surface without any interference of cell carriers such as amniotic membrane or fibrin glue. A very few studies have earlier tested the feasibility of these membranes in transplantation of limbal stem cells over the damaged corneal surfaces, which are promising in case of unilateral corneal damage [17]. In context of endogenous stem cell mobilization after injury, a few earlier studies in mice have reported a positive impact of injury on the migration of MSCs in the peripheral blood [24, 30,31]. In concordance with previous reports, we found that corneal damage creates a transient stimulus for release of MSCs from bone marrow to the peripheral blood in rabbits. Due to similar trend across mouse and rabbit species, it can be extrapolated that timing of MSC transplantation can positively affect the outcome of transplantation in humans too.

We conclusively established corneal repair after MSC transplantation using live animal OCT imaging showing smooth corneal surface and fluorescein staining showing minimal uptake of the dye. Concomitantly, the IHC of corneal sections from transplanted group clearly showed the expression of markers of corneal epithelial cells, suggesting the differentiation of rb-MSCs into corneal epithelial cells. Further the expression patterns of vimentin, collagen 1 and alpha SMA also supported the observations by showing similar expression in transplanted and normal cornea, over an extended period of time. The use of BM-MSCs in a way didn't have toxic effects on neighbouring cells and prevented any teratoma 
formation in major peripheral organs, in compliance with other reports in the literature for stem cells [32]. Further MSC transplantation didn't induce any noticeable cell death in the target tissue. Conclusions: Taken together, we observed apparently better corneal quality using temperature sensitive surfaces. The method provides distinct advantage in terms of uniformity of cell distribution. The method could be used for other types of cell transplantation over the damaged ocular surfaces.

\section{Abbreviations}

MSCs: Mesenchymal stem cells, CK3: Cytokeratin 3, a-SMA: alpha smooth muscle actin, IHC: Immunohistochemistry, LSCD: limbal stem cell deficiency, DMEM: Dulbecco's modification of Eagle medium, OCT: Optical coherence tomography, CFSE: Carboxyfluorescein succinimidyl ester

\section{Declarations}

Ethics approval and consent to participate: The study was approved by Institutional Animal Ethics Committee and the Institutional committee on Stem Cell and Research Therapy of Post Graduate Institute of Medical Education and Research (PGIMER), Chandigarh (IAEC/422 and PGI-IC-SCRT-54-2014/2981).

Consent for publication: All authors had the opportunity to see and review the manuscript, all authors give consent to publish the study.

Availability of data and material: All the data originated from the work has been put in the manuscript in the form of figures and results. The datasets used and/or analysed during the current study are available from the corresponding author on reasonable request.

Competing interests: None of the authors have competing interest.

Funding Sources: Authors would like to acknowledge Indian Council of Medical Research, New Delhi for providing personnel support to JS (3/1/3/2014/HRD-17 (10267))

Author Contributions: PG, NS and JS planned the study. PG and NS provided overall guidance in the study. JS performed majority of experiments with active help from RKS. NG helped in performing $\mathrm{IHC}$ and analysis of the same.

Acknowledgements: The authors would like to acknowledge Prof. Shobha Sehgal, department of immunopathology, PGIMER Chandigarh for help and guidance in the study. The authors would also like to acknowledge Mr Surya Prakash for support, Mr Naranjan Singh, animal attendant for help in animal handling.

\section{References}

1. Oliva MS, Schottman T, Gulati M: Turning the tide of corneal blindness. Indian J Ophthalmo/ 2012, 60(5):423-427. 
2. Ishizaki M, Zhu G, Haseba T, Shafer SS, Kao WW: Expression of collagen I, smooth muscle alphaactin, and vimentin during the healing of alkali-burned and lacerated corneas. Invest Ophthalmol Vis Sci 1993, 34(12):3320-3328.

3. Anderson DF, Ellies P, Pires RT, Tseng SC: Amniotic membrane transplantation for partial limbal stem cell deficiency. British Journal of Ophthalmology 2001, 85(5):567-575.

4. Geerling G, Maclennan S, Hartwig D: Autologous serum eye drops for ocular surface disorders. British Journal of Ophthalmology 2004, 88(11):1467-1474.

5. Di Girolamo N, Bosch M, Zamora K, Coroneo MT, Wakefield D, Watson SL: A contact lens-based technique for expansion and transplantation of autologous epithelial progenitors for ocular surface reconstruction. Transplantation 2009, 87(10):1571-1578.

6. Holland EJ, Mogilishetty G, Skeens HM, Hair DB, Neff KD, Biber JM, Chan CC: Systemic immunosuppression in ocular surface stem cell transplantation: results of a 10-year experience. Cornea 2012, 31(6):655-661.

7. Holan V, Trosan P, Cejka C, Javorkova E, Zajicova A, Hermankova B, Chudickova M, Cejkova J: A Comparative Study of the Therapeutic Potential of Mesenchymal Stem Cells and Limbal Epithelial Stem Cells for Ocular Surface Reconstruction. Stem Cells Transl Med 2015, 4(9):1052-1063.

8. Rohaina CM, Then KY, Ng AM, Wan Abdul Halim WH, Zahidin AZ, Saim A, Idrus RB: Reconstruction of limbal stem cell deficient corneal surface with induced human bone marrow mesenchymal stem cells on amniotic membrane. Transl Res 2014, 163(3):200-210.

9. Gu S, Xing C, Han J, Tso MO, Hong J: Differentiation of rabbit bone marrow mesenchymal stem cells into corneal epithelial cells in vivo and ex vivo. 2009.

10. Ma Y, Xu Y, Xiao Z, Yang W, Zhang C, Song E, Du Y, Li L: Reconstruction of chemically burned rat corneal surface by bone marrow-derived human mesenchymal stem cells. Stem Cells 2006, 24(2):315-321.

11. Oh JY, Kim MK, Shin MS, Lee HJ, Ko JH, Wee WR, Lee JH: The anti-inflammatory and anti-angiogenic role of mesenchymal stem cells in corneal wound healing following chemical injury. Stem Cells 2008, 26(4):1047-1055.

12. Jiang TS, Cai L, Ji WY, Hui YN, Wang YS, Hu D, Zhu J: Reconstruction of the corneal epithelium with induced marrow mesenchymal stem cells in rats. Molecular vision 2010, 16:1304-1316.

13. Jiang $Y$, Jahagirdar BN, Reinhardt RL, Schwartz RE, Keene CD, Ortiz-Gonzalez XR, Reyes M, Lenvik T, Lund T, Blackstad $\mathrm{M}$ et al: Pluripotency of mesenchymal stem cells derived from adult marrow. Nature 2002, 418(6893):41-49.

14. Sonmez B, Beden Ü: Fibrin glue-assisted sutureless limbal stem cell transplantation surgery for the treatment of severe ocular chemical injury. Cornea 2011, 30(3):296-300.

15. Gomes JA, dos Santos MS, Cunha MC, Mascaro VL, Barros Jde N, de Sousa LB: Amniotic membrane transplantation for partial and total limbal stem cell deficiency secondary to chemical burn. Ophthalmology 2003, 110(3):466-473. 
16. Meller D, Pires RT, Mack RJ, Figueiredo F, Heiligenhaus A, Park WC, Prabhasawat P, John T, McLeod SD, Steuhl KP et al: Amniotic membrane transplantation for acute chemical or thermal burns. Ophthalmology 2000, 107(5):980-989; discussion 990.

17. Nishida K, Yamato M, Hayashida Y, Watanabe K, Maeda N, Watanabe H, Yamamoto K, Nagai S, Kikuchi A, Tano $Y$ et al: Functional bioengineered corneal epithelial sheet grafts from corneal stem cells expanded ex vivo on a temperature-responsive cell culture surface. Transplantation 2004, 77(3):379-385.

18. Rama P, Bonini S, Lambiase A, Golisano O, Paterna P, De Luca M, Pellegrini G: Autologous fibrincultured limbal stem cells permanently restore the corneal surface of patients with total limbal stem cell deficiency1. Transplantation 2001, 72(9):1478-1485.

19. Watson SL, Marcal H, Sarris M, Di Girolamo N, Coroneo MT, Wakefield D: The effect of mesenchymal stem cell conditioned media on corneal stromal fibroblast wound healing activities. Br J Ophthalmol 2010, 94(8):1067-1073.

20. Soleimani M, Nadri S: A protocol for isolation and culture of mesenchymal stem cells from mouse bone marrow. Nat Protoc 2009, 4(1):102-106.

21. Goodell MA: Stem cell identification and sorting using the Hoechst 33342 side population (SP). Current protocols in cytometry 2005.

22. Tandon R, Gupta N, Kalaivani M, Sharma N, Titiyal JS, Vajpayee RB: Amniotic membrane transplantation as an adjunct to medical therapy in acute ocular burns. British Journal of Ophthalmology 2011, 95(2):199-204.

23. Szurman P, Warga M, Grisanti S, Roters S, Rohrbach JM, Aisenbrey S, Kaczmarek RT, Bartz-Schmidt KU: Sutureless amniotic membrane fixation using fibrin glue for ocular surface reconstruction in a rabbit model. Cornea 2006, 25(4):460-466.

24. Ye J, Yao K, Kim JC: Mesenchymal stem cell transplantation in a rabbit corneal alkali burn model: engraftment and involvement in wound healing. Eye (Lond) 2006, 20(4):482-490.

25. Reinshagen H, Auw-Haedrich C, Sorg RV, Boehringer D, Eberwein P, Schwartzkopff J, Sundmacher R, Reinhard T: Corneal surface reconstruction using adult mesenchymal stem cells in experimental limbal stem cell deficiency in rabbits. Acta Ophthalmol 2011, 89(8):741-748.

26. Hayashida Y, Nishida K, Yamato M, Watanabe K, Maeda N, Watanabe H, Kikuchi A, Okano T, Tano Y: Ocular surface reconstruction using autologous rabbit oral mucosal epithelial sheets fabricated ex vivo on a temperature-responsive culture surface. Invest Ophthalmol Vis Sci 2005, 46(5):1632-1639.

27. Gomes JA, Geraldes Monteiro B, Melo GB, Smith RL, Cavenaghi Pereira da Silva M, Lizier NF, Kerkis A, Cerruti $\mathrm{H}$, Kerkis I: Corneal reconstruction with tissue-engineered cell sheets composed of human immature dental pulp stem cells. Invest Ophthalmol Vis Sci 2010, 51(3):1408-1414.

28. Shimizu T, Yamato M, Kikuchi A, Okano T: Cell sheet engineering for myocardial tissue reconstruction. Biomaterials 2003, 24(13):2309-2316.

29. Yang J, Yamato M, Kohno C, Nishimoto A, Sekine H, Fukai F, Okano T: Cell sheet engineering: recreating tissues without biodegradable scaffolds. Biomaterials 2005, 26(33):6415-6422. 
30. Lan Y, Kodati S, Lee HS, Omoto M, Jin Y, Chauhan SK: Kinetics and function of mesenchymal stem cells in corneal injury. Invest Ophthalmol Vis Sci 2012, 53(7):3638-3644.

31. Ye J, Lee SY, Kook KH, Yao K: Bone marrow-derived progenitor cells promote corneal wound healing following alkali injury. Graefes Arch Clin Exp Ophthalmol 2008, 246(2):217-222.

32. Lian Q, Lye E, Suan Yeo K, Khia Way Tan E, Salto-Tellez M, Liu TM, Palanisamy N, El Oakley RM, Lee EH, Lim B: Derivation of clinically compliant MSCs from CD105+, CD24- differentiated human ESCs. Stem Cells 2007, 25(2):425-436.

\section{Tables}

Table 1: Description of animal groups

\begin{tabular}{|l|l|l|}
\hline S.NO. & GROUP & DESCRIPTION \\
\hline 1 & Control group $(n=3)$ & Normal cornea and bone marrow harvesting \\
\hline 2. & Damage group $(n=3)$ & Cornea damage \\
\hline 2 & $\begin{array}{l}\text { Injury + MSC transplantation } \\
(\text { Fibrin glue -15,30 and 60 days; } n=9)\end{array}$ & $\begin{array}{l}\text { Live imaging \& Eye Enucleation to evaluate corneal } \\
\text { repair and reconstruction }\end{array}$ \\
\hline 3 & $\begin{array}{l}\text { Injury + MSC transplantation } \\
(\text { Temperature sensitive membrane -15,30 } \\
\text { and 60 days; } n=9)\end{array}$ & $\begin{array}{l}\text { Live imaging \& Eye Enucleation to evaluate corneal } \\
\text { repair and reconstruction }\end{array}$ \\
\hline
\end{tabular}

\section{Figures}



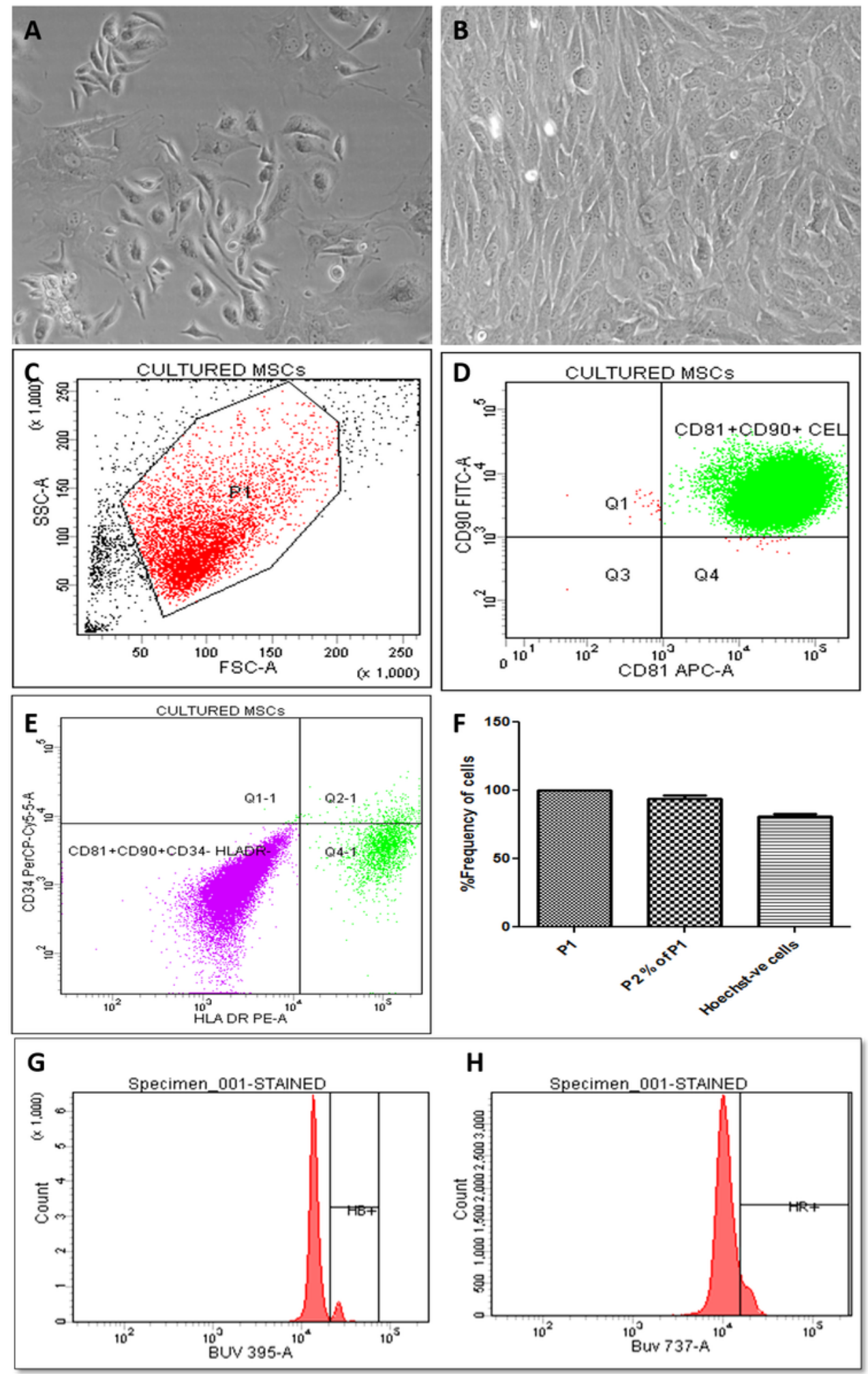

\section{Figure 1}

Culture and characterization of bone marrow derived MSCs: MSCs were cultured using explant based approach in low glucose DMEM containing $1 \%$ penstrept and $10 \%$ FBS. MSCs showed colony formation and characteristic spindle shaped morphology after 5 days of culture (A), while confluency was attained at day 14 (B). Flow cytometric analysis of MSCs upon immunostaining with CD90, CD81, CD34 and HLADR antibodies was done by gating cluster of cells P1 (C) and assessing the proportion of CD81+CD90+ 
cells gated from singlets after comparison with fluorescence minus one control (D). CD 34- HLADR- cells were gated for assessment of final phenotype of cells (E). MSCs expressed CD90 and CD81 but they didn't express CD34 and HLA-DR. Further, Hoechst blue fluorescence was detected using 424/44 filter (F), while red fluorescence was detected using 620 LP filter (G) showing MSC pumping out Hoechst dye. The percentage purity of MSCs obtained from cultures was $94.13 \pm 2.03 \%(H)$.

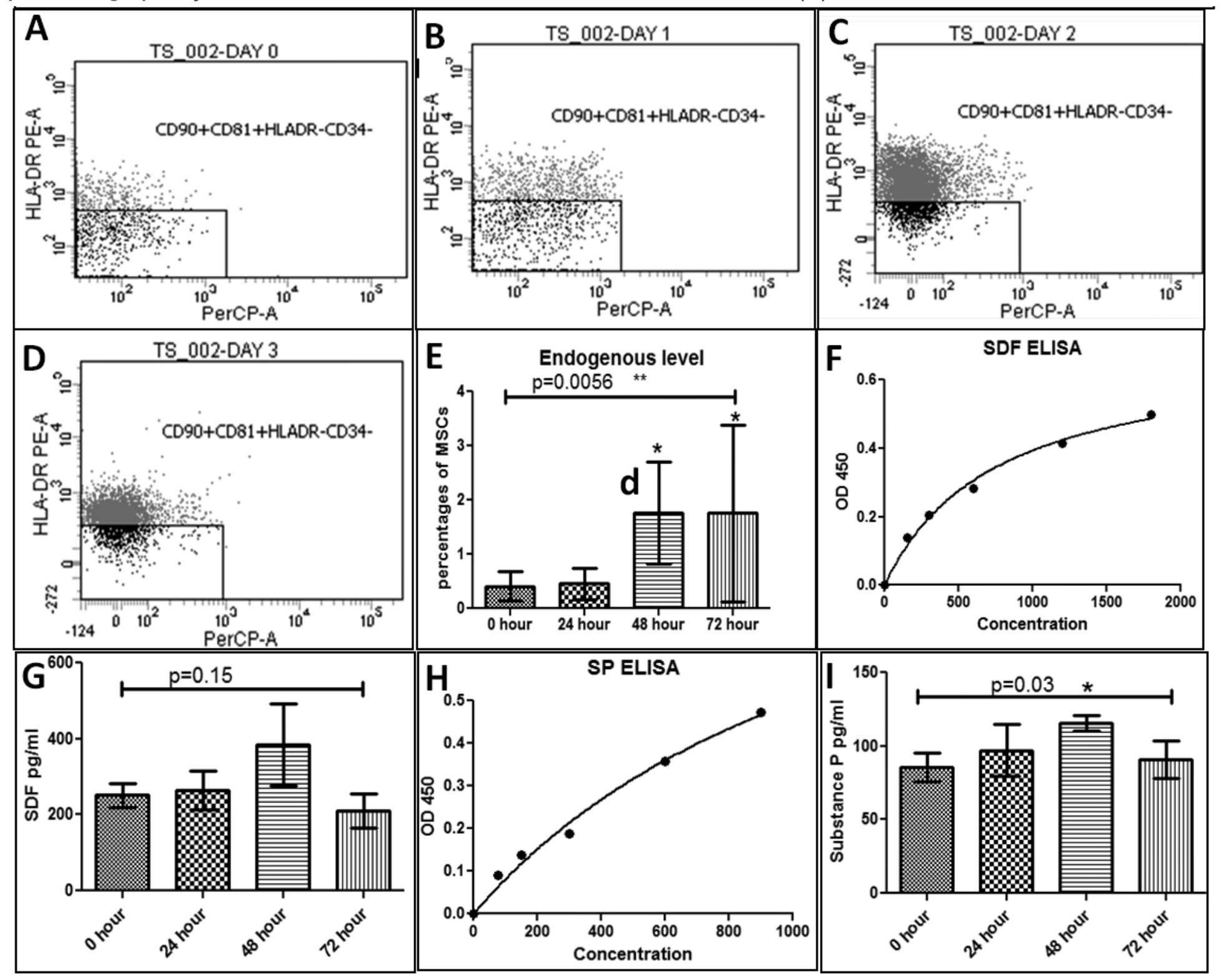

Figure 2

Effect of corneal injury on migration of MSCs: The frequency of CD90+CD81+CD34-HLA-DR- MSCs was assessed at various time intervals ranging from $0 \mathrm{hr}(\mathrm{A}), 24 \mathrm{hr}(\mathrm{B}), 48 \mathrm{hr}(\mathrm{C})$ and $72 \mathrm{hr}$ (D) after corneal injury. Frequency of MSCs in peripheral blood was significantly higher $48(p=0.0019, n=8)$ and 72 hours $(p=0.038, n=8)$ after injury, as compared to that before injury $(E)$. The levels of SDF-1a and substance $P$ were calculated from respective standard curves $(F, H)$. SDF-1 a levels were found to be apparently higher, 48 hours post injury $(384.1 \pm 54.32 \mathrm{pg} / \mathrm{ml})$ as compared to levels before injury $(252.3 \pm 15.68 \mathrm{pg} / \mathrm{ml})$ 
$(p=0.059, n=4)(G)$. Substance $P$ levels were found to be significantly higher 48 hours post corneal injury as compared to levels before injury $(p=0.03, n=4)(I)$.

\section{Figure 3}

Transplantation of MSCs over damaged corneal surface: The extent of corneal damage was assessed using corneal surface fluorescein staining. Rabbit normal cornea (A), damage cornea at day 0 (B), damage cornea at day 60 (C) and reconstructed cornea using temperature sensitive membranes at day 60 (D). H\&E stained normal cornea showed all the five layers of healthy cornea (E), while damaged cornea showed partial or complete absence of corneal epithelium, migration of leucocytes and presence of RBCs (F). Representative figure shows fibrin glue based MSC transplantation (G) and temperature sensitive membrane assisted transplantation over the damaged corneal surface as imaged at the end of the procedure $(\mathrm{H})$. The spread of transplanted cells over the damaged surface was assessed using confocal microscopy. Fibrin glue assisted transplanted eyes showed patches of CFSE fluorescence (I). The temperature sensitive membrane assisted animals showed uniform distribution of MSCs $(\mathrm{J})$.

\section{Figure 4}

Evaluation of corneal quality after MSC transplantation: The extent of repair was compared between transplanted and damaged cornea using visual corneal grading. The grades of MSC transplanted corneas using fibrin glue method were found to be significantly lower than damaged cornea $(p=0.007$, $n=9)(A)$. Similar trend was observed for MSC transplanted corneas using temperature sensitive method $(p=0.008, n=9)(B)$. Apparently higher grading scores were observed in fibrin glue method as compared to temperature sensitive method $(p=0.07, n=9)(C)$, indicating better results with the latter method. The anterior segment OCT imaging in damaged cornea showed irregularities in corneal surface as earlier as day 15 (D), while corneal ulcers at day 60 (G). MSC transplantation using temperature sensitive membranes showed smooth corneal surface at day $15(E), 60(F)$. Similarly fibrin glue MSC transplantation appeared to improve the corneal surface at day $15(\mathrm{H})$ and day $60(\mathrm{I})$. H\&E staining of damaged cornea showed absence of corneal epithelium, infiltration of leucocytes and presence of RBCs $(\mathrm{J})$, while the transplanted cornea showed re-epithelialization of cornea, post transplantation in both temperature sensitive approach (K) and fibrin glue method (L). Immunohistochemistry (IHC) of damaged cornea showed complete absence of corneal epithelium as seen by CK3 expression ( $M)$, while reepithelialization was observed after MSC transplantation using temperature sensitive membrane method $(\mathrm{N})$, as well as fibrin glue mediated transplantation (O).

\section{Figure 5}


Evaluation of corneal transparency post MSC transplantation: The evaluation of corneal quality was done using $\mathrm{IHC}$ for vimentin, a-smooth muscle actin and collagen 1. Normal cornea (A), damaged cornea (B) and the transplanted cornea (C) showed the observable differences in expression of vimentin. Similarly a-SMA expression in normal cornea (D), damaged corneas $(E)$ and transplanted cornea $(F)$ was shown. Collagen 1 expression in normal cornea $(\mathrm{G})$, damaged cornea $(\mathrm{H})$ and MSC transplanted cornea (I) also showed differences in expression. IHC scoring of vimentin levels showed statistically significant increase in expression upon chemical induced damage $(p=0.013, n=3)$, while MSC transplantation resulted in a statistically significant decrease in compared to damaged corneas, $(p=0.02, n=3)(J)$. Similarly increase in expression of $a-S M A$ after corneal damage $(p=0.002, n=3)$ compared to control cornea and decreased expression after transplantation was observed $(p=0.007, n=3)(K)$. Although, Corneal damage resulted in an insignificantly increased expression of collagen $1(p=0.10, n=3)$, statistically significant increase was observed in transplanted cornea as compared to damage alone group $(p=0.04, n=3)(L)$. A statistically significant increase in expression of collagen 1 was observed in transplanted group as compared to control, thereby signifying MSC induced wound healing $(p=0.008$, $\mathrm{n}=3)(\mathrm{L})$.

\section{Figure 6}

The effect of MSC transplantation on teratoma formation: H\&E staining of kidney (A) and liver (B) sections showed no teratoma formation. Apoptosis evaluation using TUNEL assay in control cornea showed no apoptotic nuclei (C), as compared to positive control cornea (generated using TACS nuclease) which showed many apoptotic nuclei (D). No apoptotic nuclei were observed in MSC transplanted cornea (E).

\section{Supplementary Files}

This is a list of supplementary files associated with this preprint. Click to download.

- CorneaARRIVEGuidelinesChecklistFeb2019.docx 